\title{
Characterizing naval team readiness through social network analysis
}

Jan Maarten Schraagen \& Wilfried Post 


\section{Overview}

> Team performance measurement

Social network analysis

> Case study in naval teamwork

> Conclusions, lessons learned and recommendations 


\section{Team performance measurement}

1 Huge progress made over the past decades (e.g., Brannick, Salas, \& Prince, 1997; Flin, O'Connor, \& Crighton, 2008)

1 Current team performance measurement characterized by:

$>$ Need for experienced raters

$>$ Need for multiple raters

> Need for well-calibrated raters

> Use of abstract rating categories, not always well-understood by subject-matter experts

> Constructs derived from individual approach to team cognition

$>$ Lack of specificity in terms of diagnosing deficiencies in teamwork 


\section{Team model 1}

\section{Team model 2}

/ Static team entities ('leadership';

> Dynamic team processes

'situation awareness'; 'decision

making')

> Aggregation of individual

1 Analysis at the team level knowledge

> Context-independent

> Context-dependent

1 Better teamwork leads to team effectiveness (causal I-P-O model)

> Better teamwork is an adaptive response whenever team goals are jeopardized (emergent model) 


\section{Social Network Analysis}

/ Starts with sociomatrix defining which units have a 'communicates with' relationship (e.g., Pfautz \& Pfautz, 2009; Wasserman \& Faust, 1994)

> Study real-time team interaction at the team level (Walker et al., 2006)

> Advantages:

$>$ Not dependent on availability of trained raters

> Enables precise diagnostics at specific moments in time

> Highly suitable for assessing teamwork within Team model 2 framework (Cooke et al., 2013) 


\section{Social Network Analysis}

> Base unit: communication from <actor $>$ to <actor $>$

SNA metrics used:

$>$ Degree centralization

> Eigenvector centralization

> Closeness centralization

$>$ Density

> Betweenness centralization

> Hierarchy (Krackhardt)
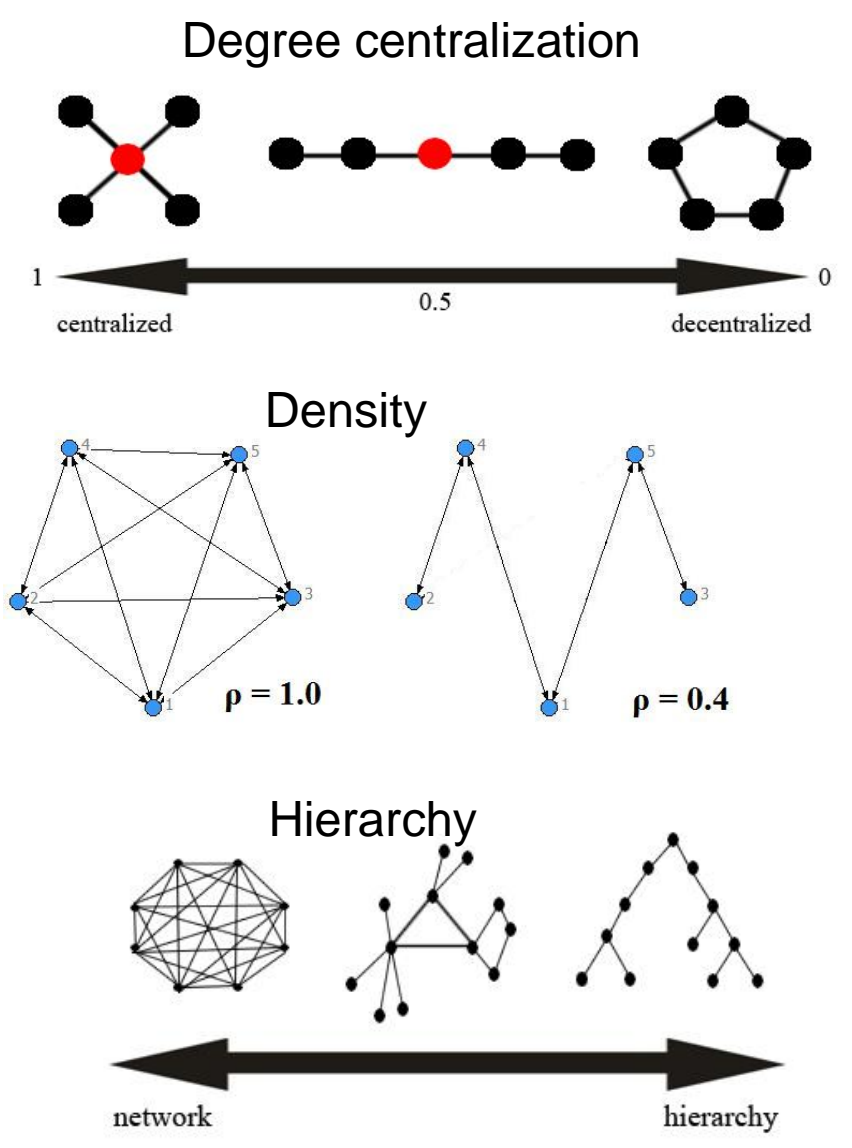


\section{Current study: naval team readiness}

/ Used Social Network Analysis techniques to study communication and coordination at the team level (ORA: Carley \& Reminga, 2004)

> Distinguished between different levels of naval team readiness 1. 'unpracticed team'

2. 'team in training'

> Research question: can we characterize naval team readiness efficiently by looking at real-time team interaction? 


\section{Method}

Observations of two Internal Battle coordination teams (5 officers each)

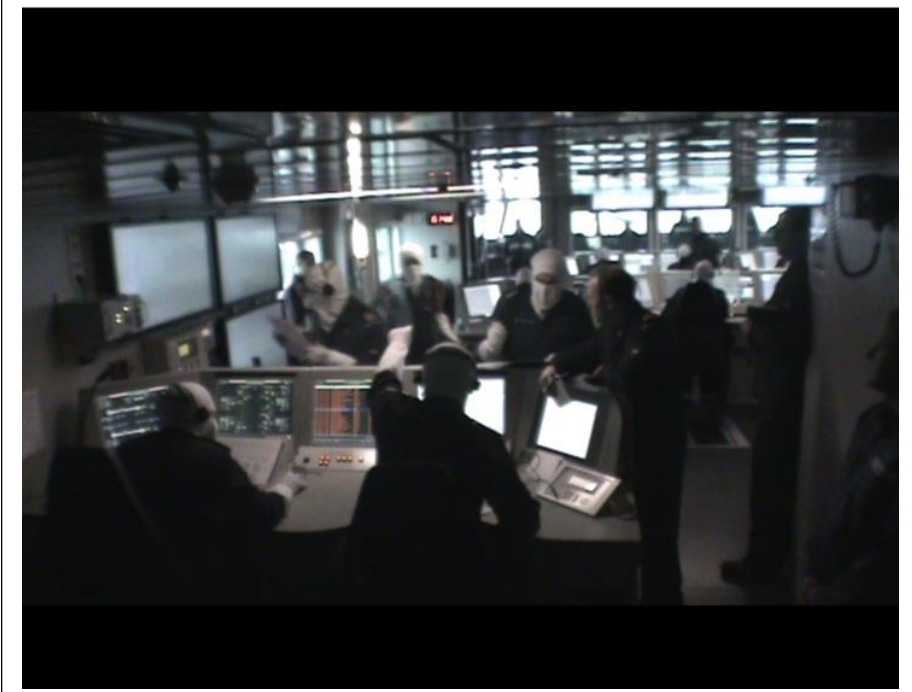

Each team: Resource Manager assisted by Damage, Sewaco, Mobility, and Personnel officers

Two highly demanding scenarios requiring all personnel on station and all systems available

Task of IB team: build adequate damage assessment within 8 minutes 


\section{Results}

\begin{tabular}{|l|c|c|}
\hline Network level measure & Unpracticed & In training \\
\hline Density & 0.80 & 1.00 \\
\hline Betweenness centralization & 0.15 & 0.50 \\
\hline Degree centralization & & \\
\hline Eigenvector centralization & 0.34 & 0.62 \\
\hline Closeness centralization & 0.26 & 0.74 \\
\hline Hierarchy & 0.25 & 0.96 \\
\hline
\end{tabular}


Sensitivity analysis, extending to actors beyond Internal Battle team

\begin{tabular}{|l|c|c|}
\hline Network level measure & Unpracticed & In training \\
\hline Density & 0.17 & 0.22 \\
\hline Betweenness centralization & 0.16 & 0.07 \\
\hline Degree centralization & & \\
\hline Eigenvector centralization & 0.16 & 0.17 \\
\hline Closeness centralization & 0.60 & 0.73 \\
\hline Hierarchy & & \\
\hline
\end{tabular}




\section{Network structures of unpracticed team (left)} versus 'team in training' (right)
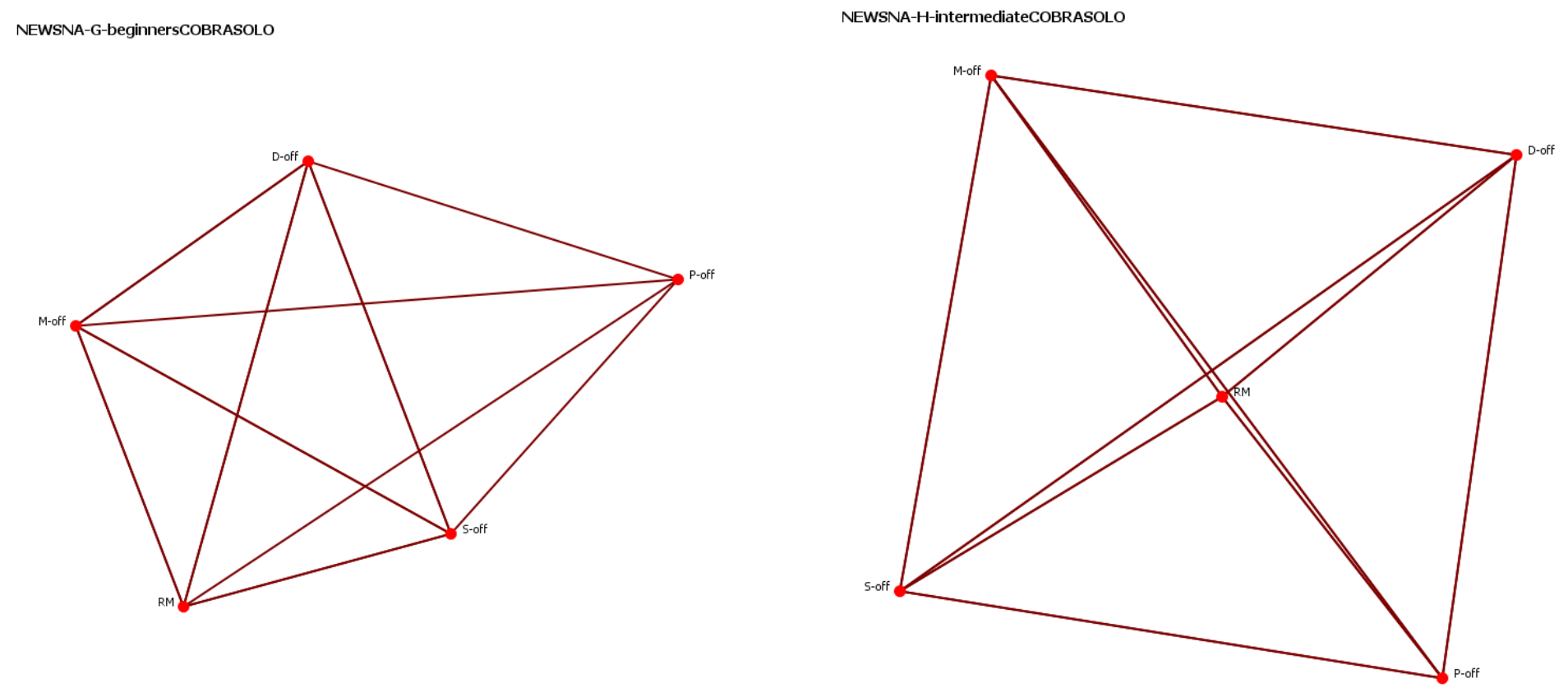
Difference scores on node level measures for RM versus average of S-, M-, D-, and P-officers on 'unpracticed' and 'in training' vessels.

\begin{tabular}{|l|c|c|}
\hline Node level measure & Unpracticed & In training \\
\hline Degree centrality & 0.25 & 0.46 \\
\hline In-degree centrality & 0.20 & 0.46 \\
\hline Out-degree centrality & 0.19 & 0.46 \\
\hline Eigenvector centrality & 0.19 & 0.56 \\
\hline
\end{tabular}




\section{Conclusions}

> Network level: More experienced team showed higher levels of information sharing and team member participation

$>$ Node level: Resource Manager played more central role in more experienced team

> Resource Manager 'in the know', needs to advice Commanding Officer

> 'Team in training' was more 'ready' than 'unpracticed' team 


\section{Lessons learned (data analysis)}

> Include core team only

> Restrict communication to actor-initiated communication (rather than proceduralized communication)

> Exclude broadcasted communication directed at groups 


\section{Recommendations and future steps}

I SNA highly suitable for point-to-point communication

> May be carried out in real time, using keyword recognition

> Useful for debriefing teams, providing objective and to the point feedback

For more information, please contact: jan_maarten.schraagen@tno.nl 
